\title{
INTERFACES ENTRE HISTORIA DE MATEMÁTICAS Y ENSEÑANZA A TRAVÉS DE ANTIGUOS INSTRUMENTOS MATEMÁTICOS: UNA EXPERIENCIA EN LA INVESTIGACIÓN ACADÉMICA
}

\author{
Ana Carolina Costa Pereira \\ carolina.pereira@uece.br \\ Universidade Estadual do Ceará
}

Recibido: 19/11/2019 Aceptado: 22/01/2020

\begin{abstract}
Resumen
Los estudios que cubren la relación entre Historia y Educación Matemática han suscitado varios debates, entre ellos, sus diferentes perspectivas pedagógicas y didácticas vinculadas a las posibles formas de abordarlas en el aula. Los vínculos entre historia, enseñanza y aprendizaje pueden ofrecer recursos que reflejarán directamente la forma de concebir ciertos conceptos matemáticos. Entre algunas posibilidades, consideramos que el estudio de los aspectos teóricos y prácticos del conocimiento y los procedimientos involucrados en la construcción y uso de instrumentos matemáticos antiguos puede llevar al estudiante a comprender no solo el proceso de producción de conocimiento, sino también la formulación de conceptos matemáticos. Así, basado en una perspectiva historiográfica actualizada, este artículo es el resultado de la pasantía postdoctoral relacionada con el proyecto "Construcción de interfaces entre la historia de las matemáticas y la enseñanza a través de instrumentos matemáticos antiguos para la elaboración de una propuesta didáctico-pedagógica dirigida a la enseñanza de conceptos matemáticos en educación básica ", que tenía como objetivo principal investigar el instrumento científico en la articulación entre la historia de las matemáticas y la enseñanza con el fin de debatir y reflexionar, así como investigar, sobre las potencialidades didácticas de un antiguo instrumento matemático. Con este fin, proponemos presentar un informe centrado en la investigación, la experiencia docente y las propuestas teóricas desarrolladas en la pasantía postdoctoral en la Pontificia Universidad Católica de São Paulo (PUCSP) en el Programa de Estudios de Postgrado en Educación Matemática supervisado por el Prof. Dr. Fumikazu.
\end{abstract}

Palabras clave: Interfaz entre la historia y la enseñanza de las matemáticas. Instrumentos matemáticos. Petrus Ramus personal.

\section{INTERFACES BETWEEN HISTORY OF MATHEMATICS AND TEACHING THROUGH OLD MATHEMATICAL INSTRUMENTS: AN EXPERIENCE IN ACADEMIC RESEARCH}

\begin{abstract}
Studies covering the relation between History and Mathematical Education have raised several debates, among them, their different pedagogical and didactic perspectives linked to the possible ways of approach in the classroom. The articulations between history, teaching, and learning can offer means that will reflect directly on how certain mathematical concepts are conceived. Among some possibilities, we consider that the study of theoretical and practical aspects of knowledge and procedures involved in the construction and use of old mathematical instruments may lead the student to understand not only the process of production of knowledge, but also the formulation of mathematical concepts. Thus, based on an updated historiographical
\end{abstract}


perspective, this article is the result of the postdoctoral training course concerning the project "Building Interfaces between the History of Mathematics and Teaching through Old Mathematical Instruments for the Development of a Didactic-Pedagogical Proposal Teaching Mathematical Concepts in Basic Education "whose main objective was to investigate the scientific instrument in the articulation between the history of Mathematics and teaching in order to discuss and reflect, as well as to investigate, the didactic potentialities of an ancient mathematical instrument. To this end, we propose to present a report focusing on research, teaching experience and theoretical propositions developed in the postdoctoral internship at the Pontifical Catholic University of São Paulo (PUCSP) in the Program of Postgraduate Studies in Mathematical Education supervised by Prof. Dr. Fumikazu.

Keywords: Interface between history and math education. Mathematical instruments. Baculum of Petrus Ramus.

\title{
INTERFACES ENTRE HISTÓRIA DA MATEMÁTICA E ENSINO POR MEIO DE ANTIGOS INSTRUMENTOS MATEMÁTICOS: UMA EXPERIÊNCIA EM PESQUISA ACADÊMICA
}

\begin{abstract}
Resumo
Estudos abrangendo as relações entre História e Educação Matemática vêm levantando diversos debates, dentre eles, suas diferentes perspectivas pedagógicas e didáticas ligadas aos possíveis caminhos de abordagem em sala de aula. As articulações entre história, ensino e aprendizagem podem oferecer recursos que irão refletir diretamente no modo de conceber certos conceitos matemáticos. Dentre algumas possibilidades, consideramos que o estudo de aspectos teóricos e práticos dos conhecimentos e dos procedimentos implicados na construção e no uso de antigos instrumentos matemáticos pode levar o discente compreender não só o processo da produção do saber, mas também da formulação de conceitos matemáticos. Dessa forma, baseado em uma perspectiva historiográfica atualizada, este artigo é resultado do estágio de pós-doutorado referente ao projeto "Construção de interfaces entre história da matemática e ensino por meio de antigos instrumentos matemáticos para a elaboração de uma proposta didático-pedagógica voltada para o ensino de conceitos matemáticos na educação básica" que tinha como objetivo principal investigar o instrumento científico na articulação entre história da Matemática e ensino com vistas a discutir e refletir, bem como investigar, sobre as potencialidades didáticas de um instrumento matemático antigo. Para tanto, propomos aqui apresentar um relato enfocando a pesquisa, experiência docente e proposições teóricas desenvolvidas no estágio pós-doutoral na Pontifícia Universidade Católica de São Paulo (PUCSP) no Programa de Estudos Pós Graduados em Educação Matemática supervisionado pelo Prof. Dr. Fumikazu.
\end{abstract}

Palavras-chave: Interface entre história e ensino de matemática. Instrumentos matemáticos. Báculo de Petrus Ramus. 


\section{Introdução}

Muitas tentativas de aproximar história e ensino de matemática podem ser encontradas em trabalhos nacionais e internacionais ${ }^{1}$. Tais iniciativas têm por base a ideia de que a história da matemática pode fornecer subsídios para desenvolver estratégias atreladas ao ensino de matemática, uma vez que trata o conhecimento matemático no seu processo de construção. Este estudo que advém de um estágio pós-doutoral também partiu deste pressuposto no momento de sua elaboração, contudo, diferentemente das muitas propostas já apresentadas, buscamos aqui aproximar história e ensino por meio da construção de interfaces, tendo por foco a fabricação e o uso de instrumentos históricos sob uma perspectiva historiográfica atualizada.

O interesse de desenvolver esta pesquisa no Programa de Estudos Pós-Graduados em Educação Matemática da PUCSP se deu por duas razões. A primeira porque, dentre os pesquisadores brasileiros que desenvolvem estudos voltados para a interface entre história e ensino de matemática, encontramos o Fumikazu Saito, professor doutor, pesquisador junto ao Programa e líder do Grupo de Pesquisa em História e Epistemologia na Educação Matemática (HEEMa). Desde 2008, ele vem se dedicando ao estudo do papel da história da matemática no ensino. Sua proposta tem por base uma historiografia atualizada da história da matemática ${ }^{2}$ que, articulada às atuais tendências da didática matemática, propicia a construção de interfaces entre história e ensino, com vistas não só a desenvolver atividades ${ }^{3}$ voltadas para o ensino de conteúdos matemáticos, mas também promover um rico diálogo entre historiadores e educadores para refletir sobre os critérios e as razões do processo da elaboração do conhecimento matemático.

A segunda razão foi de ordem didática. Em 2011, a partir de um minicurso ministrado pelos professores Fumikazu Saito e Marisa da Silva Dias no IX Seminário Nacional de História da Matemática, intitulado "Articulação de entes matemáticos na construção e utilização de instrumentos de medida do século XVI", o tema tornou-se bastante relevante por introduzir novas questões de ordem não só histórica, mas também didática ligada à articulação entre história e ensino de matemática.

\footnotetext{
${ }^{1}$ Vide, estudos de Tzanakis et al (2002), Miguel; Miorim (2004), Mendes (2009) e Chaquiam (2017).

2 Para maiores detalhes sobre as tendências historiográficas tradicional e atualizada, veja Saito (2015).

${ }^{3}$ Sobre a noção própria de interface, cf. Alfonso-Goldfarb (2003). No que diz respeito à construção de interfaces entre história da ciência e ensino, vide: Beltran (2009), Trindade et al (2010), Beltran, Saito e Trindade (2014). No que se refere à construção de interfaces entre história da matemática e ensino, vide: Saito e Dias (2013) e Saito (2016).
} 
Essas questões direcionaram nosso interesse para o mesmo tema de pesquisa do professor Saito. Desse modo, o delineamento inicial tinha o propósito de compreender o instrumento matemático na articulação entre história e ensino da matemática com vistas a discutir e refletir, bem como investigar, sobre suas potencialidades didáticas. A proposta inicial tinha em vista o estudo dos instrumentos científicos em geral, mas delimitamos para o estudo dos instrumentos matemáticos, especialmente o báculo de Petrus Ramus, como discorremos mais adiante. Os objetivos delineados no projeto inicial foram contemplados: 1) mapeamento dos diferentes significados, terminologias, tipos e usos de instrumentos ao longo da história; 2) discussão e reflexão sobre uma possível articulação entre a história e o ensino de matemática voltado para o uso de instrumentos históricos; 3) reflexão sobre as potencialidades didáticas do instrumento no ensino de matemática tendo em vista a elaboração de atividades orientadas para a Educação Básica; e 4) construção de uma interface que propicie a elaboração de propostas de ensino que contribua para a formação de conceitos matemáticos a partir do estudo de instrumento.

Dessa forma, para esse artigo dividimos a escrita em três partes. Na primeira, é apresentada, de forma sucinta, a proposta inicial da pesquisa desenvolvida. Na segunda parte, o detalhamento do que foi realizado e resultados, ou seja, os resultados da pesquisa. Por fim, algumas produções resultantes da pesquisa, em que se apresenta as atividades desenvolvidas, os trabalhos apresentados em eventos e as publicações resultantes, assim como outras pesquisas que emergiram desse processo acadêmico.

\section{Relacionando a história e o ensino da matemática por meio de uma interface}

Por interface entendemos um "conjunto de ações e de produções que promova a reflexão sobre o processo histórico da construção do conhecimento matemático com vistas a elaborar atividades didáticas que busquem articular história e ensino de matemática" (SAITO, DIAS, 2013, p. 92). Por meio dela, buscamos promover o diálogo entre historiadores, educadores e professores de matemática com o propósito de refletir sobre uma possível metodologia de abordagem voltada para a história e o ensino de matemática.

Tal diálogo, como delineamos inicialmente, tem por base um documento histórico, que pode ser um texto ou excerto de um texto, ou ainda um instrumento, um monumento, uma foto, 
uma imagem, uma figura, um vídeo, entre muitos outros. É a partir da escolha consciente do documento histórico, ou do seu consequente estudo e análise, que o diálogo tem início.

A escolha deste documento tem em vista a realização de dois movimentos: "o movimento do pensamento na formação do conceito matemático" e "o contexto no qual o conhecimento é desenvolvido" buscam alinhar as questões de ordem historiográfica da história da matemática às outras didáticas e/ou pedagógicas do ensino de matemática. Segundo Pereira e Saito (2018a, p. 4):

\begin{abstract}
O primeiro está relacionado com o pensamento na formação do conceito matemático. Trata-se de buscar no processo histórico o movimento do pensamento da apreensão do objeto e, portanto, do desenvolvimento do conceito. Esse movimento, que tem por pressuposto o objeto matemático em formação, permite que a formação de ideias componha a lógica do movimento do pensamento. Contudo, para que o lógico não prevaleça sobre o epistemológico e os fundamentos da matemática sobre a própria matemática e suas aplicações, prima-se na construção da interface a busca pelo contexto de formação desses objetos, evitando-se anacronismos e a sobreposição de temas históricas aos propósitos do ensino.

Assim, o segundo movimento se refere ao contexto no qual os conhecimentos matemáticos foram desenvolvidos, isto é, procura observar agora o conteúdo matemático, método e os motivos por trás da escrita do documento, contextualizando na época em que foi elaborado e, portanto, considerando todas as características de ordem matemática, técnica e epistemológica como propõe uma historiografia contemporânea.
\end{abstract}

Ressaltamos que são existe uma ordem para executar esses dois movimentos, pois dependerá da forma pela qual a construção da interface será conduzida por quem está à frente do processo. Dessa forma, quando o educador matemático inicia o estudo do documento histórico ele já estabelece alguns requisitos que permitem dialogar na interface, proporcionando uma ação que procura determinar relações com a elaboração de algum conceito nele retratado. Baseado nisso, começam a surgir algumas inquirições de ordem didática, pedagógica, epistemológica e matemáticas (conceituais) capaz de mostrar aspectos potencialmente didáticos e/ou pedagógicos.

A atividade resultante da interface, "busca refletir o processo da produção do conhecimento que, dependendo da intencionalidade do educador, poderá ser orientada para diferentes propostas de ensino" (SAITO; DIAS, 2013, p. 101) e está relacionada a três etapas inter-relacionadas: o tratamento didático do documento; a intencionalidade e plano de ação; e o desenvolvimento ${ }^{4}$.

\footnotetext{
${ }^{4}$ Sobre a proposta de articulação entre história e ensino de matemática na construção de interfaces, vide Saito e Dias (2013), Saito (2016) e Pereira e Saito (2018a).
} 
O tratamento didático do documento segundo Pereira e Saito (2019a, p. 347)

\begin{abstract}
está relacionado à forma pela qual o documento será apresentado ao docente e/ou discente, levando-se em consideração o objetivo que se tem em utilizá-lo, o público-alvo e o tempo disponível para o desenvolvimento da atividade por meio dele, entre outros aspectos de ordem didática.
\end{abstract}

Essa etapa é importante, pois enfatiza a tradução do documento, o uso das imagens e/ou esquemas, a importância de termos da época da escrita, assim como, as expressões e/ou os nomes de objetos inseridos. Entretanto, a intencionalidade "está ligada aos propósitos de aprendizagem e, portanto, relacionada ao momento da organização da atividade proposta. Nesta etapa, o que está em questão é o olhar do pesquisador para a potencialidade didática no intuito de articulá-la com o ensino de algum conceito matemático" (PEREIRA; SAITO, 2019a, p. 348). Isso direciona a construção de um plano de ação, ou seja, o planejamento da aplicação das atividades que será executada na sala de aula. É nesse momento que será organizado as ações que conduzirão a prática, que vai desde a entrega dos documentos históricos e/ou instrumento matemático, escolha do local para a realização das atividades, disposição e distribuição de materiais, postura do professor e dos alunos, até a escolha do tipo de organização dos grupos que melhor proporcione uma discussão mais aprofundada da temática estudada.

Por fim, o desenvolvimento é o momento que acontece a aplicação propriamente dita. Segundo Pereira e Saito (2019a, p. 348) "buscam-se considerar todas as variáveis delineadas no plano de ação de modo a propor o ensino do conceito (ou algo relacionado a ele), bem como investigar sobre os processos de ensino e de aprendizagem que emergiram da interface entre história e ensino de matemática”.

Dessa forma, visando um estudo que possibilitasse a construção de uma interface entre a história e o ensino de matemática, traçamos algumas metas, tais como integrar e consolidar a linha de pesquisa "História, Epistemologia e Didática da Matemática” e do HEEMa, por meio da vertente "Construção de interfaces entre história, ensino e aprendizagem da matemática, explorando as potencialidades didáticas de antigos instrumentos matemáticos"; fortalecer as relações acadêmicas já existentes entre o Grupo de Pesquisa em Educação e História da Matemática - GPEHM/UECE, Programa de Pós-Graduação em Ensino de Ciências e Matemática - PPGECM/IFCE e o Programa de Pós-Graduação em Educação Matemática PPGEM/PUCSP; de modo a: 1) produzir e organizar um banco de dados com as dissertações e teses produzidas na área, em particular, que envolva o estudo de instrumentos matemáticos 
voltados para o ensino; 2) colaborar com o estudo da arte de pesquisas relacionadas a antigos instrumentos matemáticos e sua articulação com a história da matemática e o ensino; e 3) publicitar a pesquisa estudada por meio de artigos em periódicos, participação em eventos e publicação de livros.

Relatamos que todas as metas foram realizadas satisfatoriamente, incluindo as atividades destinadas a elas, e que a pesquisa foi realizada a contento, perfazendo as fases da construção de uma interface entre história e ensino, a partir do instrumento matemático denominado báculo, exposto no capítulo 9 do tratado de Petrus Ramus intitulado Via regia ad geometriam - They Way of Geometry (1636).

\section{Detalhamento da realização e resultados da pesquisa}

Esta pesquisa alinhou-se às propostas que buscam construir uma interface entre história e ensino de matemática, e que são desenvolvidas pelo Grupo de Pesquisa em História e Epistemologia na Educação Matemática (HEEMa) da PUCSP, no contexto do projeto "Construção de interfaces entre história, ensino e aprendizagem da matemática, explorando as potencialidades didáticas de antigos instrumentos matemáticos". O HEEMa tem o intuito de discutir e refletir sobre as potencialidades pedagógicas da História da Matemática, investigando as muitas iniciativas de educadores e suas abordagens, tanto na pesquisa em educação matemática, quanto nas práticas docentes.

Periodicamente, no HEEMa, desenvolve seminários pesquisa entre os pesquisadores e discentes da pós-graduação em Educação matemática da PUC/SP e de demais instituições com intuito de gerar discussões sobre temáticas estudadas por seus membros de forma a contribuir com as produções científicas e outros materiais, por meio de dissertações, teses, artigos, livros, entre outros, cujo enfoque é para a elaboração de novas estratégias de ensino.

Assim, inicialmente participamos de algumas reuniões do HEEMa como forma de discutir a base teórica de nosso estudo, conjuntamente com o supervisor de estágio pós-doutoral, Prof. Dr. Fumikazu Saito. Essas discussões foram sobre os instrumentos matemáticos na história, sobre tratados de geometria prática do século XVI, e sobre a teoria que envolve a interface entre história e ensino de matemática.

Realizamos assim, um estudo inicial sobre a história dos instrumentos matemáticos no contexto de uma historiografia da história da matemática mais atualizada. Este estudo teve por 
base, inicialmente, um tratado intitulado Scripta Clarissimi Mathematici (Figura 1), publicado em 1469. A escolha deste tratado foi motivada pelo fato de ele trazer descrito o radius astronomicus, um dos muitos instrumentos matemáticos utilizados naquele período por astrônomos.

Figura 1 - Capa do Scripta clarissimi mathematici... de 1544

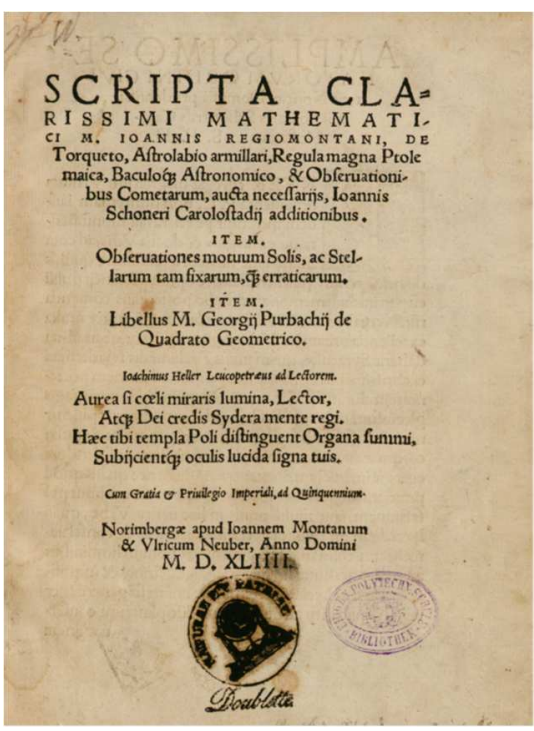

Fonte: Schöner (1544, capa)

Esses estudos tiveram como resultado dois trabalhos que foram apresentados e publicados nos anais de dois eventos. O primeiro, intitulado "O Tratado Scripta Clarissimi Mathematici (1469): uma possibilidade de uso envolvendo instrumentos", foi apresentado no 5o Simpósio Internacional de Pesquisa em Educação Matemática. Nele tratamos de apresentar e descrever a obra que reúne vários instrumentos tais como Torquatun, Astrolábio Armilar, Régua Ptolomaica e raio astronômico (PEREIRA; SAITO, 2018b).

O segundo trabalho, intitulado "Algumas breves considerações sobre o radius astronomicus (Figura 2) na interface entre história e ensino de matemática", foi apresentado no V Seminário de Escritas e Leituras em Educação Matemática. Este trabalho buscou identificar algumas potencialidades didáticas que emergiram de um estudo preliminar sobre um instrumento matemático, denominado radius astronomicus, que foi apresentado por Johann Miller (Regiomontanus), em sua obra intitulada Cometae magnitudine, longitudinecque, ac de loco eius vero Problemata XVI, publicada em 1531 (PEREIRA; SAITO, 2018c).

Figura 2 - Báculo no tratado Cometae magnitudine, longitudinecque, ac de loco eius vero Problemata XVI contida na obra Scripta clarissimi mathematici..., de 1544. 


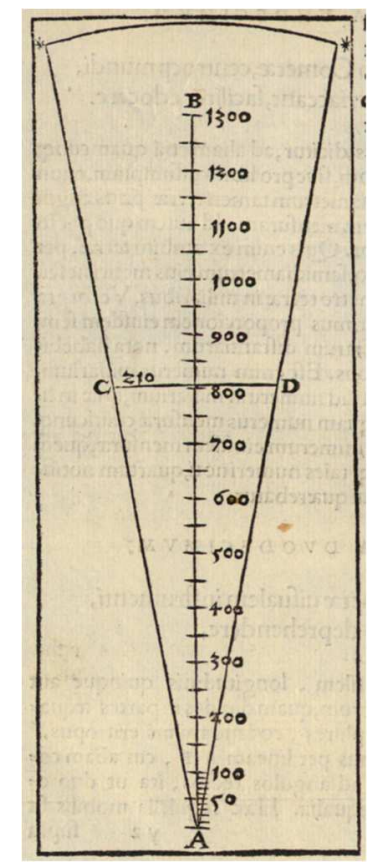

Fonte: Schöner (1544, f. 35r).

Esses dois estudos iniciais tiveram por foco, basicamente, algumas questões de ordem epistemológica e histórica de tratado que envolvessem instrumentos. Assim, ainda decorrente desses estudos iniciais, começamos a delinear um trabalho, intitulado "Um estudo preliminar sobre o Radius Astronomicus de Regiomontanus", para o GT 05 da SBEM - Sociedade Brasileira de Educação Matemática, que foi aceito e está no prelo.

Esse trabalho enfoca a contextualização histórica do instrumento radius astronomicus descrito por Johann Miller, o Regiomontanus, no opúsculo Cometae magnitudine, longitudinecque, ac de loco eius vero Problemata XVI, detendo-nos mais especificamente no problema doze que apresenta instruções acerca de sua construção e de seu uso. A edição consultada é a exposta no Scripta Clarissimi Mathematici, escrito, por volta de 1469 e publicado, em latim, no ano de 1544, sob a direção de Johannes Schöner, que traz uma compilação de tratados dos famosos astrônomos e matemáticos da época.

Também foi realizado, durante a elaboração desses trabalhos, um levantamento de estudos cuja a temática envolvia os instrumentos matemáticos na interface entre história e ensino de matemática, oriundos de pesquisas publicadas em dissertações e teses de programas de pósgraduação no Brasil. Essa compilação foi apresentada e publicada no III Seminário Cearense de História da Matemática num trabalho intitulado "Os instrumentos matemáticos na interface entre história e ensino de matemática: compreendendo o cenário nacional nos últimos 10 anos". 
Essa ação teve o propósito de produzir e organizar um banco de dados com artigos, dissertações e teses produzidas na área, em particular, que envolva o estudo de instrumentos matemáticos voltados para o ensino. Esse material foi compilado e está divulgado no site do $\mathrm{HEEMa}^{5}$ e do GPEHM $^{6}$. (PEREIRA; SAITO, 2018a).

Após esse estudo inicial, que teve por objetivo introduzir para as discussões sobre a construção de uma interface entre a história e o ensino da matemática, buscamos selecionar um instrumento que tivesse sido descrito num dos muitos instrumentos matemáticos que circularam nos séculos XVI e XVII. Escolhemos, assim, o báculo de Petrus Ramus (1515-1572) descrito no tratado intitulado Via regia ad geometriam - They Way of Geometry, edição inglesa de 1636, com o intuito de elaborar uma proposta didático-pedagógica voltada para o ensino de conceitos matemáticos na educação básica. Esse tratado foi originariamente incorporado na obra intitulada Arithmeticae libri duo: geometriae septem et viginti, publicada, em 1569, na Basiléia, Suíça. Posteriormente, foi traduzido para a língua inglesa por William Bedwell (1561 - 1632), com o título Via Regia ad Geometriam - The Way of Geometry (Figura 3), e publicado separadamente em 1636, em Londres ${ }^{7}$.

Figura 3 - Frontispício de Via Regia dd Geometriam - The Way To Geometry (1636)

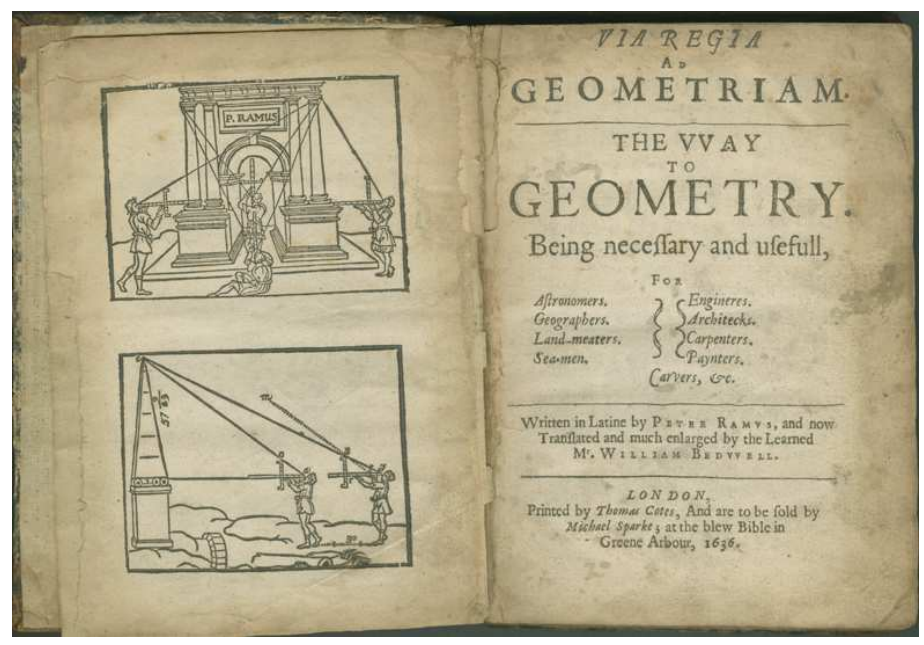

Fonte: Ramus (1636, frontispício)

\footnotetext{
${ }^{5}$ Para maiores informações acesse: https://heemaweb.wordpress.com/.

${ }^{6}$ Para maiores informações acesse: http://gpehm.blogspot.com/.

${ }^{7}$ Para o desenvolvimento do estudo utilizamos a versão inglesa Ramus (1636) que foi cotejada com outras duas versões latinas, Ramus $(1569,1599)$.
} 
Assim, foi realizado um estudo contextual e historiográfico do tratado, Via regia ad geometriam - They Way of Geometry (1636), em que ministramos um seminário de pesquisa no HEEMa apresentando algumas considerações iniciais sobre de Petrus Ramus (1515-1572) e sua obra que faz parte da rica literatura dedicada à geometria prática publicada ao longo dos séculos XVI e XVII.

As discussões e os debates durante o seminário revelaram que grande parte da audiência, composta basicamente por discentes do Programa de Estudos Pós-Graduados em Educação Matemática (PPGEM/PUCSP), não conhecia a importância de Petrus Ramus e sua obra para o desenvolvimento da geometria prática do século XVI, assim como o instrumento denominado de báculo pelo autor.

Isso, entretanto, não nos causou espanto, visto que Ramus é pouco conhecido na matemática pelos historiadores no cenário nacional e internacional, mas teve um impacto considerável no que diz respeito à reforma curricular das universidades em seu período. Ele se envolveu ativamente no combate contra o aristotelismo e a escolástica cultivado dentro das universidades, defendendo que todo ensino deveria ser vinculado a prática, em que os alunos poderiam compreender com mais facilidade e naturalidade. Esse pensamento é o que ele chamava de "método" que consistia em um meio de ensino eficaz, em que trazia seus alunos para uma compreensão profunda do conteúdo, organizando sempre os conceitos mais gerais para os mais específicos (GOULDING, 2006). Segundo Pereira e Saito (2018a, p. 27) "na opinião de Ramus, a única forma de colocar as matemáticas em seu verdadeiro caminho era valorizando um ensino que primasse pela utilidade do conhecimento matemático, ou seja, que tivesse por base a aplicação de teoremas a problemas práticos".

Com relação ao tratado de Ramus, o estudo teve por base a tradução inglesa que foi publicado por William Bedwell (1561-1632) e cotejado com a edição latina de 1569. O tratado foi editado por Jonh Clerke (?1596 - 1658), em que na nota para o leitor, ele informa que a tradução apresentada foi realizada inicialmente por Thomas Hood (1556-1620), mas nunca publicada anteriormente com demonstrações e diagramas ${ }^{8}$. No prefácio, Bedwell também faz menção à primeira versão publicada por Hood e observa que fez algumas alterações em sua tradução, mudando algumas expressões e inserindo novas informações aos quais são

\footnotetext{
${ }^{8}$ Cf. Ramus (1590).
} 
acrescentados comentários e outras observações que elucidam alguns conhecimentos geométricos presente na obra.

A obra trata de uma das muitas compilações que buscaram organizar diferentes conhecimentos geométricos que transitaram em distintos segmentos de saber desde a Antiguidade Clássica. Orientado para resolver questões de ordem prática, seu conteúdo parece à primeira vista um conjunto desordenado de proposições geométricas quando comparado aos Elementos de Euclides.

As primeiras impressões deste estudo inicial da obra de Ramus foi apresentada no $8^{\circ}$ Encontro Luso-Brasileiro de História da Matemática. Com o título "Um estudo preliminar da obra Via Regia ad Geometriam (1636) de Petrus Ramus", este trabalho teve em vista apresentar um estudo preliminar dessa obra com vistas em reconhecer aspectos que ora a aproximam e ora a afastam da tradição da geometria prática medieval. (PEREIRA; SAITO, 2018e).

O Via regia ad geometriam está organizado em vinte e sete livros. O livro que nos interessou para esta pesquisa é o nono, intitulado "O nono livro de Geometria de Petrus Ramus, que trata da medição de linhas retas por meio de triângulos retângulos semelhantes”. Dividido em dezesete tópicos ou capítulos, que vai da página 113 a 135 (vinte e duas páginas no total), este livro apresenta várias maneiras de medir "linhas retas" por meio de um instrumento matemático denominado "báculo" (Figura 4).

Figura 4 - Situação de medição em que o báculo é utilizado.

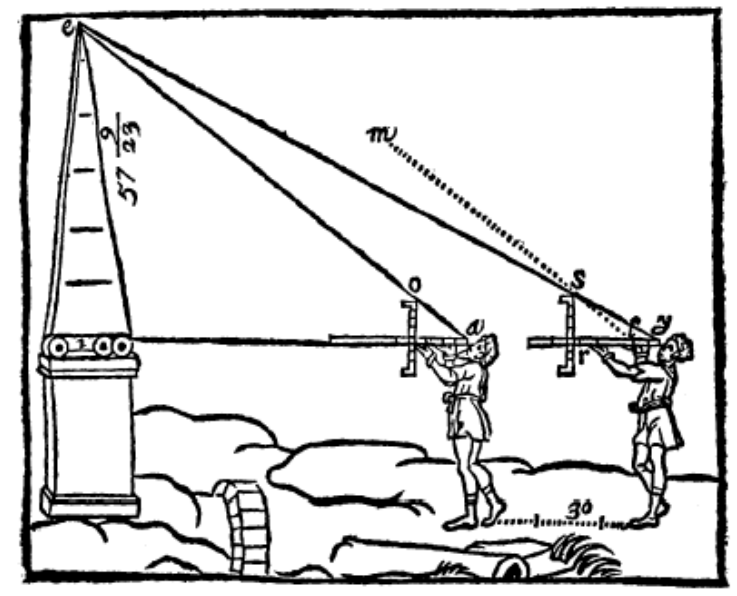

Fonte: Ramus (1636, p. 122)

Dessa forma, a partir do estudo da parte em que Ramus se refere ao báculo, buscamos percorrer os dois movimentos "o contexto no qual o conhecimento foi desenvolvido" e o 
"movimento do pensamento na formação do conceito matemático". Para tanto, traduzimos o nono livro e, a partir das orientações ali contidas, procuramos construir um protótipo do instrumento com vistas a estudá-lo e a compreendê-lo. Os principais apontamentos e outras questões de ordem teórica ligadas à construção do instrumento foram publicados na Revista Cocar, com o título "A reconstrução do báculo de Petrus Ramus na interface entre história e ensino de matemática”. (PEREIRA; SAITO, 2019a)

As questões que emergiram da construção do protótipo do báculo serviram de base para elaborarmos uma atividade que consistiu inicialmente em reconstruir o báculo de Ramus, suscitando questões de ordem matemática que poderiam ser exploradas numa situação de medida, valorizando condicionantes manipulativos, a partir de uma situação-problema. Assim, foi desenvolvida uma atividade que explorou alguns aspectos do conhecimento matemático incorporado no báculo por meio de seu uso numa situação de medição.

A atividade teve o intuito de mapear algumas potencialidades didáticas e/ou pedagógicas, que emergem do seu manuseio, a partir das ações que envolve o uso do báculo de Ramus. Cabe, entretanto, aqui observar que a atividade proposta foi um "piloto", e, portanto, consiste numa primeira tentativa de mapear as ações que ressignificam conceitos matemáticos bem elementares, utilizando antigos instrumentos matemáticos.

Desse modo, com o intuito de avançarmos no estudo de construção da interface entre história e ensino de matemática, foi planejado e aplicado um curso de extensão universitária realizado na Universidade Estadual do Ceará (UECE) para doze professores de matemática (Figura 5). Esse curso foi organizado em forma de oficinas, em quatro encontros no período matutino, com duração de aproximadamente cinco horas, de 16 a 19 de julho de $2018^{9}$. Todos os encontros foram filmados e os participantes entregaram, ao final de cada etapa um relatório, descrevendo suas impressões sobre o processo realizado e os procedimentos executados.

Figura 5 - Postura e medição com o báculo.

\footnotetext{
${ }^{9}$ Os instrumentos de coleta de dados aplicados foram autorizados pelos participantes e pelo Comitê de Ética da UECE, sob n ${ }^{\circ}$ 3.285.665 e CAAE: 08268319.7.0000.5534.
} 


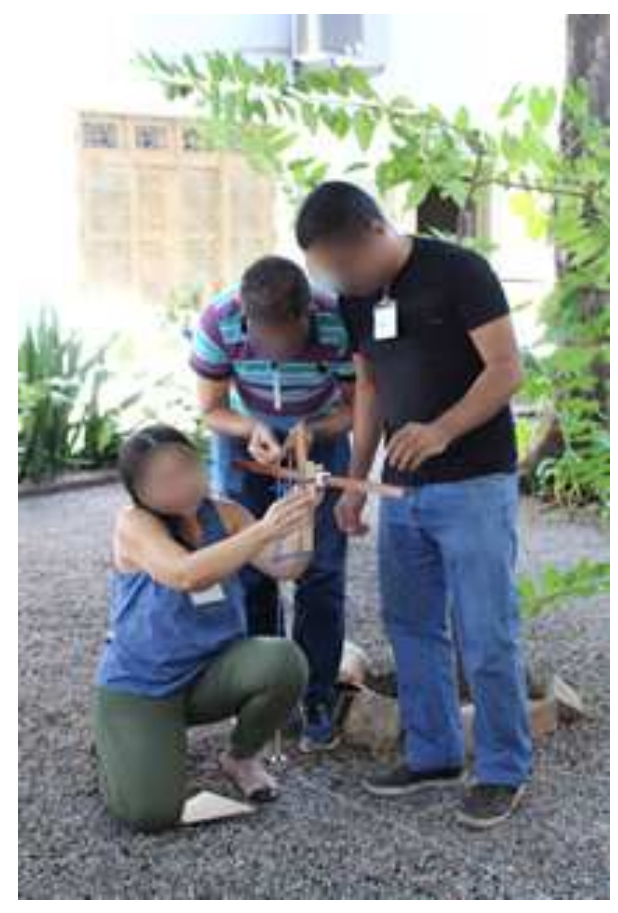

Fonte: Arquivo pessoal do autor.

Esse curso possibilitou a coleta de vários dados, em que muitos ainda precisam ser tratados. Uma parte desses resultados foi enviado para publicação na revista Educação Matemática Pesquisa da PUCSP, cujo título é "Os conceitos de perpendicularidade e de paralelismo mobilizados em uma atividade com o uso do báculo (1636) de Petrus Ramus" (PEREIRA; SAITO, 2019b). Esse artigo tem a intenção de apresentar alguns resultados preliminares de uma atividade que envolveu o uso de um instrumento denominado "báculo", descrito por Petrus Ramus (1515-1572) em sua obra intitulada Via regia ad geometriam - The Way of Geometry, com vistas a elencar algumas potencialidades didáticas do báculo. Focou-se nas propriedades geométricas deste instrumento de modo a mapear um conjunto de ações implicado no processo de medição.

Outra produção vinculada a construção da interface entre história e ensino, foi publicada na Revista de Matemática, Ensino e Cultura (REMATEC) intitulada "A organização do saber geométrico em Via Regia ad Geometriam (1636) de Petrus Ramus: uma reflexão sobre a definição de ângulo reto e de perpendicular". Neste artigo tratamos de uma discussão sobre a elaboração do tratado de geometria de Ramus e de sua relação com a reforma curricular da faculdade de artes da Universidade de Paris. Também é apresentada uma descrição do tratado expondo suas partes e sua organização, confrontando com os Elementos de Euclides; e por fim, 
é explorado um exemplo relacionado às definições de ângulo reto e de retas perpendiculares, que pode promover uma reflexão sobre a noção de perpendicularidade. Esse estudo nos deu uma visão de como os conceitos eram organizados em sua obra e como eles eram ensinados. Ramus apresenta primeiro a definição de retas perpendiculares, para posteriormente definir ângulo reto, apoiando-se em instrumentos geométricos como a régua, o compasso, o esquadro e o perpendiculum. Compreendemos que esse procedimento estava estreitamente relacionado à noção própria de geometria aqui considerada, que recorre aos instrumentos geométricos para aferir medidas, que reformulam e alteram a disposição das definições. (PEREIRA; SAITO, 2018a)

Como forma de sintetizar a produção acadêmica desenvolvida o estágio pós-doutoral, elaboramos, em conjunto com o supervisor deste estágio, um material no formato de livro que colabora para as atuais reflexões sobre a interação entre história da matemática e educação matemática. Este livro será lançado no XIII Seminário Nacional de História da Matemática em abril/2019 intitulado A elaboração de atividades com um antigo instrumento matemático na interface entre história e ensino. Neste livro, apresentamos os principais resultados das discussões, que contribuíram não só para o desenvolvimento da pesquisa, mas também para o aprimoramento das questões de ordem teórica em relação à construção interfaces entre história e ensino. Segundo Saito e Pereira (2019, p. 8) o livro visa "discutir sobre as potencialidades didáticas e/ou pedagógicas que emergem do processo de construção e de uso de um instrumento matemático denominado "báculo", descrito em Via regia ad geometriam - The Way of Geometry, publicado em 1636". É selecionado alguns excertos do documento para discutir a elaboração de atividades com um instrumento nele descrito, "com vistas a elaborar um material que constituísse uma proposta de ensino a ser desenvolvida com os professores. (SAITO; PEREIRA, 2019, p. 8)

Ainda no que diz respeito aos estudos sobre os conceitos matemáticos mobilizados em tratados, aprovamos o trabalho nomeado de "Um estudo preliminar sobre o conceito de ângulo reto em tratados de geometria prática do século XVI" para ser apresentado no XIII Seminário Nacional de História da Matemática. Esse estudo é apresentado a noção de ângulo reto, no status da geometria prática e sua relação com o conhecimento geométrico teórico e aplicado em três tratados do século XVI: The pathwaie to knowledge de Robert Recorde (1510-1558), Via regia ad geometriam - They Way of Geometry de Peter Ramus (1515-1572), e Geometrical Practise 
named Pantometria de Thomas Digges (1546-1595). Segundo os autores esse "estudo preliminar nos evidenciou que uma análise além das definições, ou seja, olhando a disposição, a organização dos teoremas e das proposições e dos problemas práticos neles expostos notamos que a exposição dos conceitos depende de outros fatores que não são matemáticos em essência" (PEREIRA; SAITO; 2019c, p. 16).

Outras atividades também foram realizadas que estão estreitamente relacionadas ao desenvolvimento desta pesquisa. Dentre elas podemos citar: a participação de algumas aulas da disciplina Tópicos de História e Filosofia da Matemática, ministrada pelo Prof. Fumikazu Saito, durante 2018.2; o convite para ministrar uma palestra no Seminário do Programa de Estudos Pós-Graduados em Educação Matemática da Pontifícia Universidade Católica de São Paulo (PUC/SP) intitulada "Regiomontanus e seu legado para o desenvolvimento de conhecimentos trigonométricos", em outubro de 2018; a participação bancas de qualificação e defesa de trabalhos de mestrado de ambas instituições (IFCE e PUCSP).

No que se refere a participação de bancas, ela visa fortalecer as relações acadêmicas já existentes entre o Grupo de Pesquisa em Educação e História da Matemática - GPEHM (UECE), Programa de Pós-Graduação em Ensino de Ciências e Matemática - PPGECM (IFCE) e o Programa de Pós-Graduação em Educação Matemática - PPGEM (PUCSP). Os temas das dissertações estão ligados diretamente a temática da pesquisa desenvolvida no estágio pósdoutoral, visto que todas estão ligadas a construção de uma interface entre história e ensino de matemática.

\section{Considerações Finais}

Desenvolver uma pesquisa acadêmica na interface entre as áreas de história da matemática e educação matemática que requer estudo teórico de um documento em seus vários aspectos, conceitual, historiográfico e epistemológico, vinculando a propostas didáticas que podem ser implantadas na educação básica ou na formação de professores, demanda tempo. Esse artigo relatou uma experiência vivenciada no estágio pós-doutoral desenvolvido no ano de 2018 com duração de 12 meses enfocando aspectos como desenvolvimento da pesquisa, experiência da docente/pesquisadora e proposições teóricas.

Percebemos que, embora haja um crescimento de pesquisa que envolvem a inserção na história da matemática no ensino no século XXI, poucas são aquelas que conseguem apresentar 
uma proposta didática que vise construir um corpo de conhecimentos matemáticos por meio da história, sob uma perspectiva historiográfica atualizada.

Os instrumentos matemáticos no seu caráter teórico e experimental estudado sob o ponto de vista do saber-fazer, torna-se um potencial elemento do docente na interface entre a história e ensino, visto que permite discutir questões epistemológicas, axiológicas e ontológicas sejam estudadas. Entretanto, para o pesquisador, é um estudo demasiadamente cansativo, pois além dos obstáculos apresentado na coleta de material documental da época da obra, envolve a tradução de documentos do século XVI, que requer cuidado, pois a linguagem, as expressões, as imagens e os esquemas são desconhecidos em contextos mais atual.

Dessa forma, esse estudo não termina aqui, outros pontos encontrados na própria obra estudada, Via regia ad geometriam - The Way of Geometry, de Petrus Ramus, seja ela envolvendo seu instrumento, o báculo, ou o conteúdo geométrico apresentado, ainda precisam ser aprofundadas. Outro ponto, são as questões metodológicas que embasam a interface entre história e ensino de matemática, pouco discutida até então.

\section{Referências}

ALFONSO-GOLDFARB, A. M. Como se daria a construção de áreas interface do saber?. Kairós. V. 6, N. 1. São Paulo: Núcleo de Estudo e Pesquisa do Envelhecimento e Programa de Estudos Pós-Graduados em Gerontologia (PUCSP), 2003. pp. 55-66.

BELTRAN, M. H. R. História da ciência e ensino: algumas considerações sobre a construção de interfaces. In: WITTER, G. P.; FUJIWARA, R. (Org.). Ensino de ciências e matemática: análise de problemas. São Paulo: Ateliê Editorial, 2009, p. 179-208.

BELTRAN, M. H. R.; SAITO, F.; TRINDADE, L. S. P. História da ciência para formação de professores. São Paulo: Ed. Livraria da Física; CAPES/OBEDUC, 2014.

CHAQUIAM, M. Ensaios Temáticos História e Matemática em sala de aula. Belém: Sbem/Sbem-pa, 2017.

GOULDING, R. Method and Mathematics: Peter Ramus's Histories of the Sciences. Journal of the History of Ideas, v. 67, n. 1, p. 63-85, 2006.

MENDES, I. A. Investigação histórica no ensino da Matemática. Rio de Janeiro: Ciência Moderna, 2009. 
MIGUEL, A.; MIORIM, M. Â. História na Educação Matemática: Propostas e desafios. Coleção Tendências em Educação Matemática. Belo Horizonte: Autêntica, 2004.

PEREIRA, A. C. C.; SAITO, F. A organização do saber geométrico em Via Regia ad Geometriam (1636) de Petrus Ramus: uma reflexão sobre a definição de ângulo reto e de perpendicular. Rematec. V. 13, N. 27. Natal: Grupo de Estudos e Pesquisas sobre Cultura Matemática e suas Epistemologias na Educação Matemática, 2018a. pp.24-38.

PEREIRA, A. C. C.; SAITO, F. A reconstrução do báculo de Petrus Ramus na interface entre história e ensino de matemática. Revista Cocar, v. 13, n. 25, pp. 342-372, 2019a.

PEREIRA, A. C. C.; SAITO, F. Algumas breves considerações sobre o Radius Astronomicus na interface entre história e ensino de matemática In: As múltiplas linguagens da educação matemática na formação e nas práticas docentes.1 ed. Fortaleza: EDUECE, 2018c, v.1, p. 699714.

PEREIRA, A. C. C.; SAITO, F. O Tratado Scripta Clarissimi Mathematici (1469): uma possibilidade de uso envolvendo instrumentos In: 5o Simpósio Internacional de Pesquisa em Educação Matemática, 2018b, Belém. Anais do 5o Simpósio Internacional de Pesquisa em Educação Matemática. Belém: SBEM-PA, 2018b. v.1. p.1 - 15.

PEREIRA, A. C. C.; SAITO, F. Os instrumentos matemáticos na interface entre história e ensino de matemática: compreendendo o cenário nacional nos últimos 10 anos In: III Seminário Cearense de História da Matemática, 2018, Juazeiro do Norte - CE. Anais do III Seminário Cearense de História da Matemática. Fortaleza: Editora da UECE, 2018d. v.1. p.1 - 12.

PEREIRA, A. C. C.; SAITO, F. Um estudo preliminar da obra Via Regia Ad Geometriam (1636) de Petrus Ramus In: 8o Encontro Luso Brasileiro de História da Matemática, 2018, Foz do Iguaçu. Caderno de Resumos do 80 Encontro Luso Brasileiro de História da Matemática. Foz do Iguaçu: UNIOESTE, 2018e. v.1. p.71 - 71.

PEREIRA, A. C. C.; SAITO, F. Um estudo preliminar sobre o conceito de ângulo reto em tratados de geometria prática do século XVI. In: SEMINÁRIO NACIONAL DE HISTÓRIA DA MATEMÁTICA, 13., 2019, Fortaleza. Anais... . Fortaleza: Sbhmat, 2019c. p. 1092 - 1107.

PEREIRA, A. C. C.; SAITO, F.. Os conceitos de perpendicularidade e de paralelismo mobilizados em uma atividade com o uso do báculo (1636) de Petrus Ramus. Educação Matemática Pesquisa, São Paulo, v. 21, n. 1, p.405-432, 2019b.

RAMUS, P. Arithmeticae libri duo: geometriae septem et vinti. Basileae: per Eusebium Episcopium \& Nicolai fratris haeredes, 1569. 
RAMUS, P. Arithmeticae libri duo: geometriae septem et vinti. Francofurti: Andreae Wecheli Heredes, Claudium Marninum \& Ioannem Aubrium, 1599.

RAMUS, P. Via Regia ad Geometriam: The way to geometry. London: Thomas Cotes, 1636.

SAITO, F. História da matemática e suas (re)construções contextuais. São Paulo: Editora Livraria da Física, 2015. 259 p.

SAITO, F. História e ensino de matemática: construindo interfaces. In: FLORES SALAZAR, J.; UGARTE GUERRA, F. (eds.). Investigaciones en Educación Matemática. Lima: Fondo Editorial PUCP, 2016, p. 237-291.

SAITO, F.; DIAS, M. S. Interface entre história da matemática e ensino: uma atividade desenvolvida com base num documento do século XVI. Ciência \& Educação, Bauru, v. 19, n. 1, p.89-111, mar. 2013. Quadrimestral.

SAITO, F.; PEREIRA, A. C. C. A elaboração de atividades com um antigo instrumento matemático na interface entre história e ensino. São Paulo: Livraria da Física, 2019. (História da Matemática e da educação matemática para o ensino, volume 2).

SCHONER, J. (Ed.) Scripta Clarissimi Mathematici M. Ioannis Regiomontani, De Torqueto, Astrolabio armillari, Regula magna Ptolemaica, Baculoque Astronomico, Obseruationibus Cometarum, aucta necessarijs, Ioannis Schoneri Carolostadij additionibus; Item. Obseruationes motuum Solis, ac Stellarum tam fixarum, quam erraticarum; Libellus M. Georgii Purbachii de Quadrato Geometrico. Nürnberg: Johann Montanus \& Ulrich Neuber 1544.

TRINDADE, L. S. P.; RODRIGUES, S. P.; SAITO, F.; BELTRAN, M. H. R. História da Ciência e Ensino: alguns desafios. In: BELTRAN, M. H. R.; SAITO, F.; TRINDADE, L. S. P. (Orgs.). História da Ciência: Tópicos Atuais. São Paulo: Ed. Livraria da Física/CAPES, 2010, p. 119-132.

TZANAKIS, C. et al. Integrating history of mathematics in the classroom: an analytic survey. IN: FAUVEL, J.; MAANEN, J. van. History in Mathematics Education: The ICMI Study. Dordrecht/Boston/London: Kluwer Academic Publishers, v. 6, 2002. p. 201- 240.

Autora

\section{Ana Carolina Costa Pereira}

Pós-doutorado em Educação Matemática pela Pontifícia Universidade Católica de São Paulo. Doutorado em Educação pela Universidade Federal do Rio Grande do Norte (2010) e Mestrado em Educação Matemática pela Universidade Estadual Paulista Júlio de Mesquita

Filho (2005). Graduação em Licenciatura em Matemática pela Universidade Estadual do Ceará (2001). Atualmente é Professora Adjunta da Universidade Estadual do Ceará e líder do Grupo de Pesquisa em Educação e História da Matemática (GPEHM). Tem experiência na 
Interfaces entre história da matemática e ensino por meio de antigos instrumentos...

área de Educação Matemática, com ênfase em História de Matemática, atuando principalmente nos seguintes temas: formação de professores de matemática e interface entre história e ensino de matemática. Mais informações no Currículo Lattes: http://lattes.cnpq.br/1062497580478584. Orcid: http://orcid.org/0000-0002-3819-2381. E-mail: carolina.pereira@uece.br 